\title{
ON VEHICLE EVALUATION AND DESIGN USING DATA ENVELOPMENT ANALYSIS WITH HIERARCHICAL CONCEPTS
}

\author{
Parque, Victor; Honobe, Kazuhiro; Miura, Satoshi; Miyashita, Tomoyuki \\ Department of Modern Mechanical Engineering, Waseda University, Japan
}

\begin{abstract}
In recent years, product complexity in terms of function and structure has been driven by technological development in complementary components. Designing unbiased product evaluation metrics being to grasp the complex relationships of product features, and able to capitalize on market needs has become a challenge in industrial practice.

In this paper, we propose a hybrid framework in which evaluation models are generated by integrating Interpretive Structural Modeling (ISM), Hierarchical Clustering and Data Envelopment Analysis (DEA). Whereas ISM constructs hierarchical digraphs (skeletons), Hierarchical Clustering reduces dimensionality of pairwise comparisons (correlations) of design variables, and suggests possible evaluation configurations, and DEA computes weights to provide optimal evaluation metrics. Our computational experiments using more than twenty thousand vehicles from 1982 to 2013 confirmed the feasibility and usefulness of DEA with hierarchical concepts to generate the optimal vehicle evaluation metric, and to suggest configurations for vehicle design layouts.
\end{abstract}

Keywords: Design informatics, New product development, Conceptual design

\section{Contact:}

Parque, Victor

Waseda University

Modern Mechanical Engineering

Japan

parque@aoni.waseda.jp

Cite this article: Parque, V., Honobe, K., Miura, S., Miyashita, T. (2019) 'On Vehicle Evaluation and Design Using Data Envelopment Analysis with Hierarchical Concepts', in Proceedings of the 22nd International Conference on Engineering Design (ICED19), Delft, The Netherlands, 5-8 August 2019. DOI:10.1017/dsi.2019.128 


\section{INTRODUCTION}

Along with the development of complementary technology, the complexity of the structure and the functionality of mechanical products have continuously expanded in recent years, e.g. vehicles as complex multi-functional systems. And along with differentiation and consumer satisfaction needs, machines have become more complex and diverse. To accurately grasp changes in diversified markets, companies have been analyzing large amounts of data to design effective R\&D policies to capitalize on the gap between consumer needs and product development. In line with the above, since the early 20 's, the use of data-driven product evaluation has attracted the attention of business stakeholders, in which weighted indexes often combine qualitative and quantitative metrics, and algorithmic techniques aid in the multi-objective optimization of the product evaluation metrics.

However, multi-objective optimization requires the definition of weights, which in the context of product design, biases towards local optima metrics (Tavana et al. (2016)). To avoid biased weights on evaluation metrics, Data Envelopment Analysis (DEA) (Charnes et al. (1978)) is a well-known nonparametric approach which computes weights based on the fact that each evaluation item gets the highest evaluation in relative terms; thus, it is possible to construct a one-dimensional indicator avoiding biases to local optima judgements (Barat et al. (2018); Forsund (2018)), which has positive implications for marketing and product development.

There exists several studies focusing on DEA, for instance, the work by (Doyle (2014)) and (Seiford and Zhu (2003)) on printers, and the work by (Papagapiou et al. (1997)) on vehicles. Also, (Papagapiou et al. (1997)) studied automobiles released in 1996, and divided the evaluation items into groups which define economic evaluation items and technical evaluation items, demonstrating the usefulness of DEA to compare and decide a particular product based on the rich information provided by DEA. Also, Gonzalez et al. (2015) studied the efficiency of 2092 cars, and found that dealers' discounts is inversely related to car efficiency. Voltes-Dorta et al. (2013) tested the ability of car manufacturers in Spain to meet $\mathrm{CO}_{2}$ emission targets with the existing technological trends.

In addition, (Fernandez-Castro et al. (2002)) have shown the consistency of DEA with consumer choice in Economics literature, and provided an example on the manageable reduction of number of diesel cars which need user assessment. Also, (Papahristodoulou (1997)) evaluated the efficiency of 121 personal cars in 1997, provided comparisons in a normative manner with economic and technical parameters. Also, (Cantner et al. (2012)) used product variables of German vehicles over the period 2001 to 2006, constructed a fitness evaluation ratio based on DEA, and found its direct relationship to market share and consistency with evolutionary theory of "growth of the fit".

The use of historical information and the use of DEA-inspired evaluation models for vehicle design offer the possibility to explore and expand towards multi-functional mobility models. In the past research, for instance, (Papagapiou et al. (1997)) has evaluated 121 car models, and (Fernandez-Castro et al. (2002)) evaluated 44 cars. Thus, DEA has been a useful method to evaluate vehicle performance using historical data, enabling the recommendation of new product layouts and product choices. However, DEA requires the definition of input and output variables in product configurations, which diminishes the effectiveness if not properly identified. Thus, in complex product configurations, it is likely to generate a large biased number of DEA evaluation models based on arbitrary user views of input-output variables.

Interpretive Structural Model (ISM) (Warfield (1973a,b, 1974a,b, 1973c, 1974c)) is a promising tool to aid in model choice and visualization of relationship among design variables, being useful to tackle the above-mentioned problem. Generally speaking, ISM constructs network structures portraying the hierarchical nature of relationships among components in a product. For instance, (Raut et al. (2017)) identified the critical success factors for adopting cloud computing in the Indian companies; and (Hsiao et al. (2013)) established the hierarchical architecture of a bicycle by using the Interpretive Structural Model (ISM), and obtained the optimal performance of the hierarchical modules after market segmentation. (Han et al. (2015)) analyzed the energy efficiency of Chinese ethylene industry by integrating the main factors for energy consumption identified by ISM, whose arbitrariness in selection is avoided, and whose efficiency is solved by slack variables in DEA. Also, a recent extension of DEA is applied to energy and environment studies (Sueyoshi et al. (2017)).

Although the integration of DEA and ISM is a potential tool to generate effective evaluation metrics in vehicle studies (Papagapiou et al. (1997); Gonzalez et al. (2015); Voltes-Dorta et al. (2013); FernandezCastro et al. (2002); Papahristodoulou (1997); Cantner et al. (2012)), there still exists gaps in model 


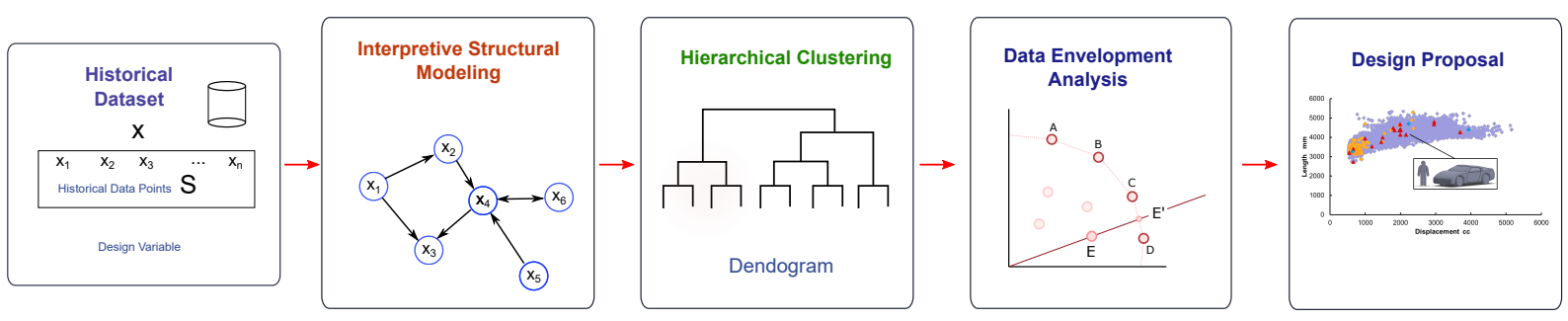

Figure 1. Basic idea of proposed approach.

selection: the large number of vehicle variables makes difficult to evaluate the entire space of configurable models. Thus, previous studies on vehicle design based on DEA metrics have focused only on a limited amount of historical information (Papagapiou et al. (1997); Gonzalez et al. (2015); Voltes-Dorta et al. (2013); Fernandez-Castro et al. (2002); Papahristodoulou (1997); Cantner et al. (2012)).

In this research, in order to fill the above-mentioned gap, we propose a new method to generate comprehensive evaluations based on the integration of Interpretive Structural Modeling, Hierarchical Clustering and Data Envelopment Analysis as shown by Figure 1. Basically, ISM constructs the hierarchical digraph (skeleton) based on relationships of vehicle variables, and Hierarchical Clustering reduces the dimensionality of pairwise comparisons (correlations) of design variables, and suggests the useful configurations of evaluation models. Finally, Data Envelopment Analysis computes the optimal weights and provides a metric to evaluate vehicle performance by considering the relationship of vehicle design variables. In particular, our contributions are as follows:

1. A hybrid framework to generate evaluation metrics based on Interpretive Structural Modeling (ISM), Hierarchical Clustering (HC) and Data Envelopment Analysis DEA.

2. Computational experiments show the feasibility of our proposed approach over 27,858 vehicle models from 1982 to 2013, containing 12 design variables, including vehicle sizing, torque, engine performance, fuel consumption, price and weight.

3. A comprehensive evaluation metric for vehicle design consisting of a hierarchical and modular digraph, as well as proposals for new vehicle design layouts with maximal performance metric.

We believe our proposed method offers the useful tools which aids in the generation of unbiased vehicle evaluation metrics and models and achieving the maximal performance frontiers. In the next sections we describe our proposed approach, as well as the computational experiments to evaluate its feasibility.

\section{PROPOSED METHOD}

\subsection{Basic idea}

The basic idea of our method is portrayed by Figure 1, which is based on the following tenets:

1. A historical dataset of design variables is known a-priori, where in $x_{1} \ldots x_{n}$ denote the set of $n$ variables within a time frame.

2. The hierarchical digraph is computed by using the Interpretive Structural Modeling, whose role is to provide a compact graph structure whose definition depicts the relationship among the design variables $x_{1} \ldots x_{n}$.

3. Hierarchical Clustering computes modules of design variables in order to reduce dimensionality of pairwise comparison of design variables.

4. Data Envelopment Analysis computes the efficiency ratio on pairwise comparisons rendered by the Hierarchical clustering.

5. New Design Proposals are suggested based on the evaluation ratio of DEA on the historical dataset.

\subsection{Interpretive structural modeling}

The Interpretive Structural Modeling (ISM) is a method proposed by Warfield aiming to represent organized knowledge in binary matrices and multilevel digraphs (Warfield (1973a,b, 1974a,b, 1973c, 1974c)), which aids to the visual expression of relationships among elements of a system through hierarchical structure and transitivity of relationships. Let $X=x_{i}$ be the set of design variables; then a boolean 
matrix $B$ is first created with $1(0)$ denoting the presence (absence) of relationship between elements in the system $X$. Then, the reachability matrix $M$, or transitive closure of $B$, is computed as follows:

$$
(B+I)^{k-1} \neq(B+I)^{k}=(B+I)^{k+1}=M,
$$

where $k$ is a positive integer which is less the number of elements in the set $X$. In the above, there is a unique reachability matrix $M$ for any square boolean matrix $B$.

On the basis of the reachability matrix $M$, if we connect and render all the elements of the matrix $M$, the network becomes complex and difficult to understand. Therefore, a skeleton matrix is created by deleting paths within a module, by which a hierarchical diagram is created (Warfield (1973b, 1974a,b)).

\subsection{Hierarchical clustering}

Hierarchical Clustering classifies computes dendrogram to group the similarity of design variables. Here, the correlation between two design variables is considered as distance between points, and the Ward's approach is considered for distance between groups.

Although it is possible to use the k-means and spectral approaches, Hierarchical Clustering is advantageous to reduce the dimensionality of pairwise comparisons and is useful to render a hierarchy of clusters in terms of correlation factors; thus its becomes possible to compute compact and highly related set of design variables.

\subsection{Data envelopment analysis}

Data Envelopment Analysis (DEA) was developed by A. Charnes and W. W. Cooper in the late 70's with the aim of comparing the efficiency of Decision Making Units (DMUs) with multiple inputs and multiple outputCharnes et al. (1978). In this method, the measure of efficiency of a DMU is computed as the maximum ratio of weighted outputs to weighted inputs subject to the condition that the upper bound of similar ratios is one. Formally,

Maximize

$$
\theta_{o}=\frac{\sum_{r=1}^{s} u_{r} y_{r o}}{\sum_{i=1}^{m} v_{i} x_{i o}}
$$

subject to

$$
\begin{aligned}
& \frac{\sum_{r=1}^{s} u_{r} y_{r j}}{\sum_{i=1}^{m} v_{i} x_{i j}} \leq 1, j=1, \ldots, m . \\
& u_{r}, v_{i} \geq 0 ; r=1, \ldots, s ; i=1, \ldots, m .
\end{aligned}
$$

where $y_{r j}$ are known outputs and $x_{i j}$ are known inputs of the $j$-th DMU; and $u_{r}, v_{i} \geq 0$ are the weights to be computed from the solution of the above problem. The efficiency $\theta_{o}$ is set relative to other DMUs, which implies that the maximization enables computing favorable weights within the allowable constraints (efficient frontier). Thus, the weight coefficients $u_{r}, v_{i}$ are set such that the evaluation value $\theta_{o}$ becomes the maximum on the condition that the evaluation value $\theta$ for $j=1, \ldots, m$ does not exceed 1. On the other hand, proposals for improvement of inefficient targets can be introduced based on relationships to the excellent group (efficient frontier). Furthermore, solutions to Eq. 2 is computable by conversion to linear programs.

\section{GENERATING EVALUATION METRICS USING VEHICLE DATA}

In order to evaluate the feasibility of our proposed method, we performed computational experiments within the context of vehicle layout design. This section describes our experimental settings, and discusses our obtained results.

\subsection{Dataset and variables}

In this study, we collected twelve design variables of 27,858 vehicle models between 1982 to 2013, whose key description is shown by Table 1 . 


\begin{tabular}{|c|c|c|c|c|c|c|c|c|c|c|c|c|c|c|c|c|c|c|c|c|c|c|c|c|}
\hline & $\begin{array}{l}0 \\
1\end{array}$ & $\begin{array}{l}0 \\
2\end{array}$ & $\begin{array}{l}0 \\
3\end{array}$ & $\begin{array}{l}0 \\
4\end{array}$ & $\begin{array}{l}0 \\
5\end{array}$ & $\begin{array}{l}0 \\
6\end{array}$ & $\begin{array}{l}0 \\
7\end{array}$ & $\begin{array}{l}0 \\
8\end{array}$ & $\begin{array}{l}0 \\
9\end{array}$ & $\begin{array}{l}1 \\
0\end{array}$ & $\begin{array}{l}1 \\
1\end{array}$ & $\begin{array}{l}1 \\
2\end{array}$ & $\begin{array}{l}1 \\
3\end{array}$ & $\begin{array}{l}1 \\
4\end{array}$ & $\begin{array}{l}1 \\
5\end{array}$ & $\begin{array}{l}1 \\
6\end{array}$ & $\begin{array}{l}1 \\
7\end{array}$ & $\begin{array}{l}1 \\
8\end{array}$ & $\begin{array}{l}1 \\
9\end{array}$ & $\begin{array}{l}2 \\
0\end{array}$ & 2 & $\begin{array}{l}2 \\
2\end{array}$ & $\begin{array}{l}2 \\
3\end{array}$ & $\begin{array}{l}2 \\
4\end{array}$ \\
\hline 01 Torque $\uparrow$ & 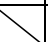 & $x$ & & & & & & & 0 & & 0 & & 0 & & & & & & & & 0 & & 0 & \\
\hline 02 Torque $\downarrow$ & $x$ & 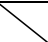 & & & & & & & & 0 & & 0 & & 0 & & & & & & & & 0 & & 0 \\
\hline 03 Maximum Output $\uparrow$ & & & 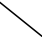 & $x$ & & & & & 0 & & 0 & & 0 & & & & & & & & 0 & & 0 & \\
\hline 04 Maximum Output $\downarrow$ & & & $x$ & 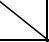 & & & & & & 0 & & 0 & & 0 & & & & & & & & 0 & & 0 \\
\hline 05 Displacement $\uparrow$ & 0 & & 0 & & 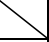 & $x$ & & & & & & & & & & & & & & 0 & & & & \\
\hline 06 Displacement $\downarrow$ & & 0 & & 0 & $\times$ & 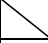 & & & & & & & & & & & & & 0 & & & & & \\
\hline 07 Length $\uparrow$ & & & & & & & 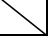 & $x$ & & & & & & & & & & & & & 0 & & 0 & \\
\hline 08 Length $\downarrow$ & & & & & & & $x$ & 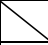 & & & & & & 0 & & & & & & & & 0 & & 0 \\
\hline 09 Width $\uparrow$ & & & & & & & & & 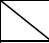 & $x$ & & & & & & & & & & 0 & 0 & & 0 & \\
\hline 10 Width $\downarrow$ & & & & & & & & & $x$ & 1 & & & & & & 0 & & & 0 & & & 0 & & 0 \\
\hline 11 Height $\uparrow$ & & & & & & & & & & & 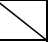 & $x$ & & & & & & & & 0 & 0 & & 0 & \\
\hline 12 Height $\downarrow$ & & & & & & & & & & & $x$ & 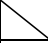 & & & & & & 0 & 0 & & & 0 & & 0 \\
\hline 13 Indoor Length $\uparrow$ & & & & & & & 0 & & & & & & 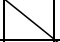 & $x$ & & & & & & & 0 & & & \\
\hline 14 Indoor Length $\downarrow$ & & & & & & & & & & & & & $x$ & 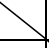 & & & & & & & & 0 & & \\
\hline 15 Indoor Width $\uparrow$ & & & & & & & & & 0 & & & & & & 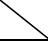 & $x$ & & & & & 0 & & & \\
\hline 16 IndoorWidth $\downarrow$ & & & & & & & & & & & & & & & $x$ & $\triangle$ & & & & & & 0 & & \\
\hline 17 Indoor Height $\uparrow$ & & & & & & & & & & & 0 & & & & & & 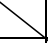 & $x$ & & & 0 & & & \\
\hline 18 Indoor Height $\downarrow$ & & & & & & & & & & & & & & & & & $x$ & 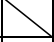 & & & & 0 & & \\
\hline 19 Fuel Consumption $\uparrow$ & & & & & & & & & & & & & & & & & & & 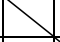 & $x$ & & & & \\
\hline 20 Fuel Consumption $\downarrow$ & & & & & & & & & & & & & & & & & & & $\times$ & $\searrow$ & & & & \\
\hline 21 Price $\uparrow$ & & & & & & & & & & & & & & & & & & & & & 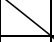 & $x$ & & \\
\hline 22 Price $\downarrow$ & & & & & & & & & & & & & & & & & & & & & $x$ & 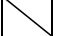 & & \\
\hline 23 Weight $\uparrow$ & 0 & & 0 & & & & & & & & & & & & & & & & & 0 & 0 & & $\searrow$ & $x$ \\
\hline 24 Weight $\downarrow$ & & & & & & & & & & & & & & & & & & & 0 & & & 0 & $x$ & $\lambda$ \\
\hline
\end{tabular}

Figure 2. Design structure matrix of vehicle layout with 24 evaluation items.

Table 1. Vehicle variables.

\begin{tabular}{cll}
\hline Variable & Description & Units \\
\hline$x_{1}$ & Total Length & $\mathrm{mm}$ \\
$x_{2}$ & Total Width & $\mathrm{mm}$ \\
$x_{3}$ & Total Height & $\mathrm{mm}$ \\
$x_{4}$ & Indoor Length & $\mathrm{mm}$ \\
$x_{5}$ & Indoor Width & $\mathrm{mm}$ \\
$x_{6}$ & Indoor Height & $\mathrm{mm}$ \\
$x_{7}$ & Torque & $\mathrm{kg} \cdot \mathrm{m}$ \\
$x_{8}$ & Maximum Output & $\mathrm{ps}$ \\
$x_{9}$ & Displacement & $\mathrm{cc}$ \\
$x_{10}$ & Fuel Consumption & $\mathrm{km} / 1$ \\
$x_{11}$ & Price & $\mathrm{JPY}$ \\
$x_{12}$ & Weight & $\mathrm{kg}$ \\
\hline
\end{tabular}

Furthermore, in order to define the relationships between variables, we divided the variables in two subtypes: one which denotes increment $(\uparrow)$, and one which denotes decrement $(\downarrow)$. Therefore, our study analyzed the relationship among variables with 24 evaluation items ${ }^{1}$.

\subsection{Interpretive structural modeling}

Pairwise comparisons are shown in Figure 2, and the hierarchical digraph (skeleton) obtained by the Interpretive Structural Modeling is shown by Figure 3-(a). By observing Figure 3-(a), we can readily note that the hierarchical digraph is roughly divided into two independent groups. Also, the observed patterns for increment and decrement between the two groups are non-repeatable, in which (01) Torque $\uparrow$, (03) Maximum Output $\uparrow$, (05) Full Length $\uparrow$, (07) Full Width $\uparrow,(09)$ Total Height $\uparrow$, and (23) Weight $\uparrow$ create a different structure. Furthermore, variations in the 24 evaluation items induces in variations of

${ }^{1} 24=12$ variables $\times 2$ instances, $\uparrow$ and $\downarrow$, per variable. 


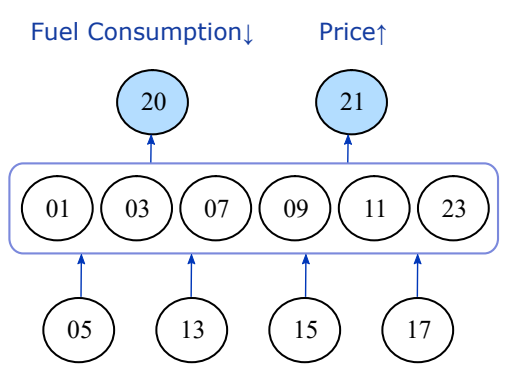

Skeleton Group A

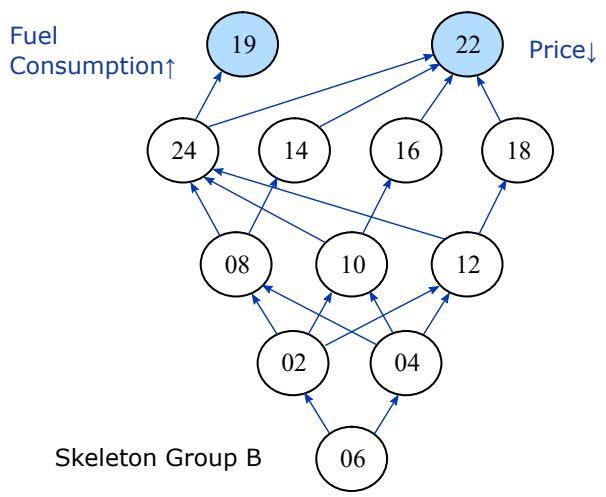

(b) Dendogram (Hierarchical Clustering)
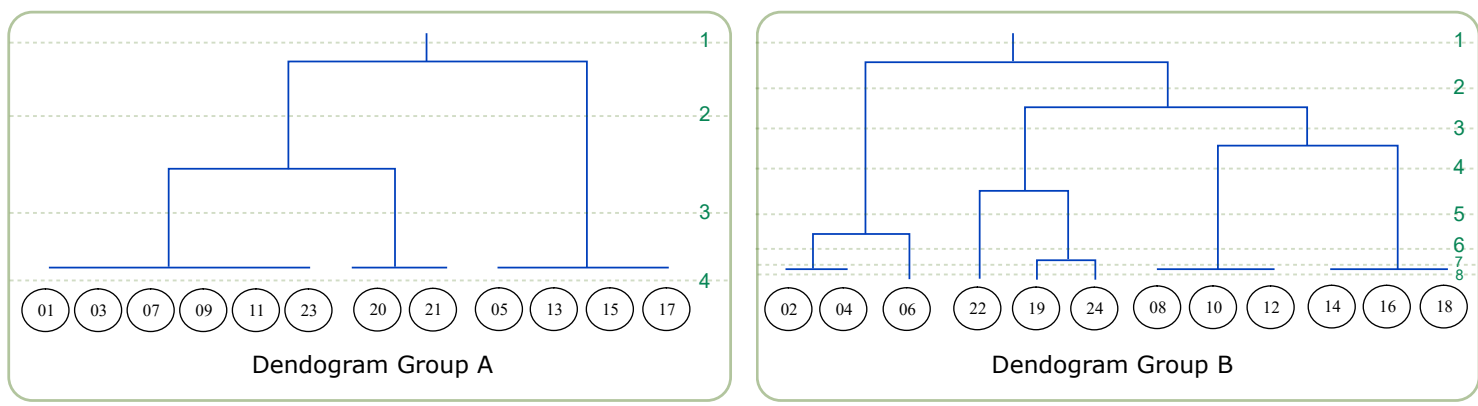

(c) Best Evaluation Model

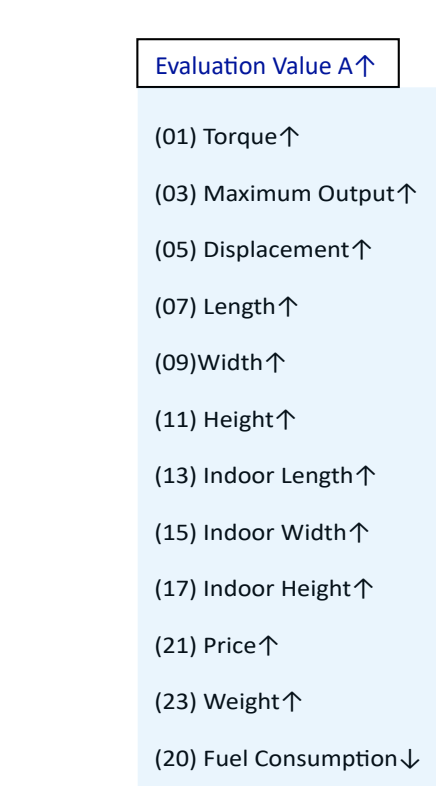

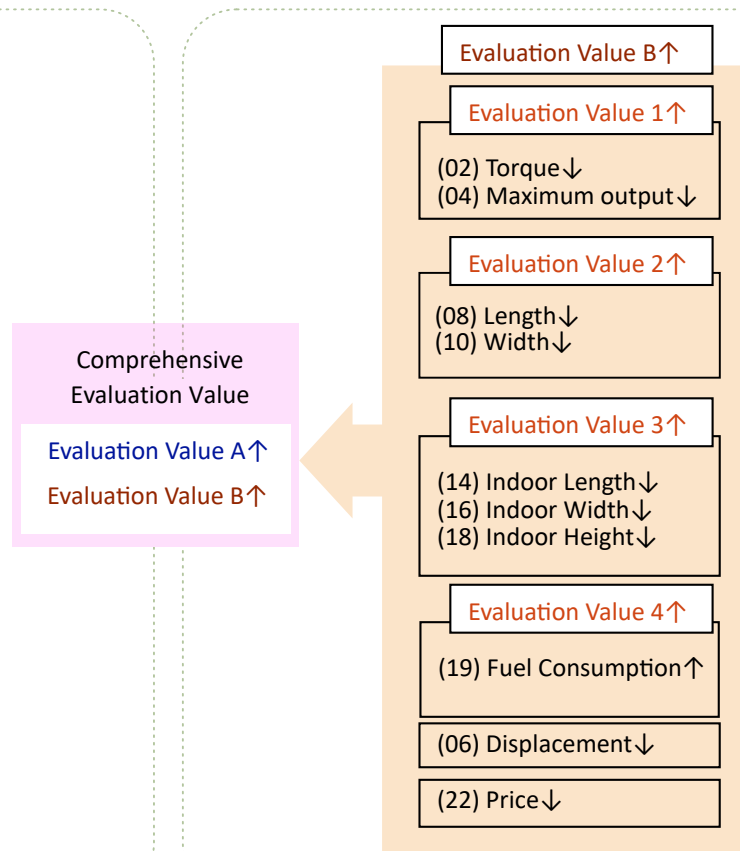

Figure 3. (a) Hierarchical digraph computed by interpretive structural modeling, (b) Dendrograms computed by hierarchical clustering, (c) Evaluation model constructed from dendrogram.

(19) Fuel Consumption $\uparrow$, (20) Fuel Consumption $\downarrow$, (21) Price $\uparrow$ and (22) Price $\downarrow$, due to being located in the upper stratum of the hierarchical digraph. For convenience, we denote the skeleton located at the left of Figure 3-(a) as Group A, and denote the skeleton located at the right of Figure 3-(a) as Group B. 


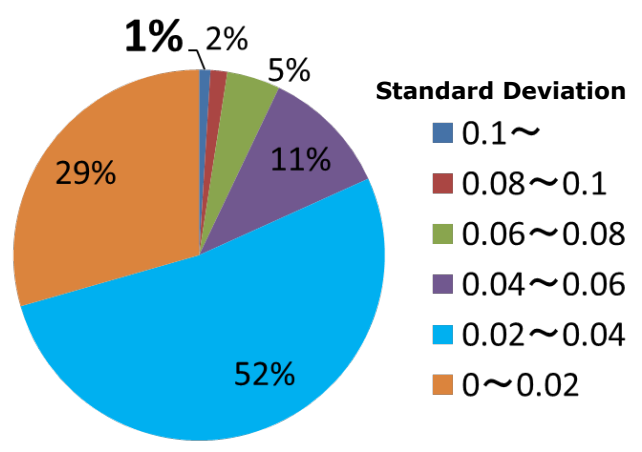

(a) DEA

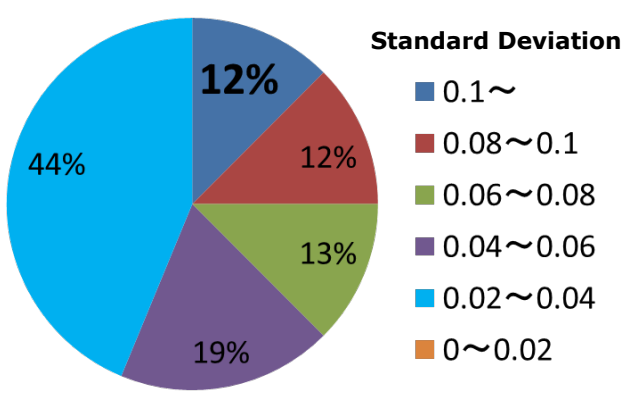

(b) Proposed Method

Figure 4. Frequency of standard deviation in (a) DEA and (b) Propose method.

\subsection{Hierarchical clustering}

Then, by using the skeletons from Figure 3-(a), dendrograms are constructed by agglomerative clustering, whose results are shown by Figure 3-(b). The shape of the dendrogram of Group A is more simple compared to that of Group B. We believe this occurs due to the fact that Group A has a hierarchical digraph with a modular component, which implies rendering a shallow dendrogram; on the other hand, the dendrogram of Group B is deeper due to the presence of a diverse set of uncorrelated of variables. In order to compute a comprehensive evaluation, all models combining all hierarchies of Group A and Group B were evaluated, and the model with the maximum standard deviation is selected (large dispersion in evaluations implies better coverage). The hierarchies in each group are computed by applying threshold cuts in the vertical axis of each dendrogram; for instance, there exists 4 types of hierarchies in Dendrogram A by applying 4 thresholds cuts (numbered-dotted-green lines) in Figure 3-(b). As a result of we evaluating $32^{2}$ models, model with maximum standard deviation of 0.110 is depicted by Figure 3-(c).

As we can readily note, Figure 3-(c) shows the following facts: (1) the evaluation in Group A consists of a single cluster with large number of increments, and the evaluation of Group B consists of a large number of clusters and decrements. (2) as for Group B, it is possible to induce in variations in the evaluation result by classifying according to characteristics such as engine performance (Torque and Maximum Output), external size (Length and Width), inner size (Indoor Length, Width and Height), Fuel Consumption, Displacement and Price.

\subsection{Comparison with DEA}

In order to evaluate the effectiveness of introducing the hierarchical concepts into the construction of models, we compared our proposed approach with that of conventional Data Envelopment Analysis (DEA), as follows: (1) The vehicle variables were classified as either input or output, and all 4096 configurations $^{3}$ corresponding to all combinations of input-output were evaluated by DEA. (2) The frequency of standard deviation comprising all models is shown by Figure 4 . We can observe the following facts:

1. DEA has less frequency of higher standard deviation compared to our proposed approach: DEA has $1 \%$ of its models having standard deviation equivalent to 0.1 or more, while $12 \%$ of our models in our proposed approach has standard deviation of 0.1 or more.

2. DEA comprises more models with less standard deviation: about $81 \%$ of its models has standard deviation of 0.04 or less, while $56 \%$ of our models has standard deviation of 0.04 or less.

The above insights have implications on the feasibility of our approach to explore the search space of robust evaluation metrics more effectively. Whereas shallow-DEA limits its search space to the configuration of design variables, the DEA with hierarchy concepts is able to expand the search space due to the combinatorial nature of modules rendered by and Hierarchical Clustering.

\footnotetext{
24 hierarchies in Group A $\times 8$ hierarchies in Group B

${ }^{3} 2^{12}=4096$ configurations
} 

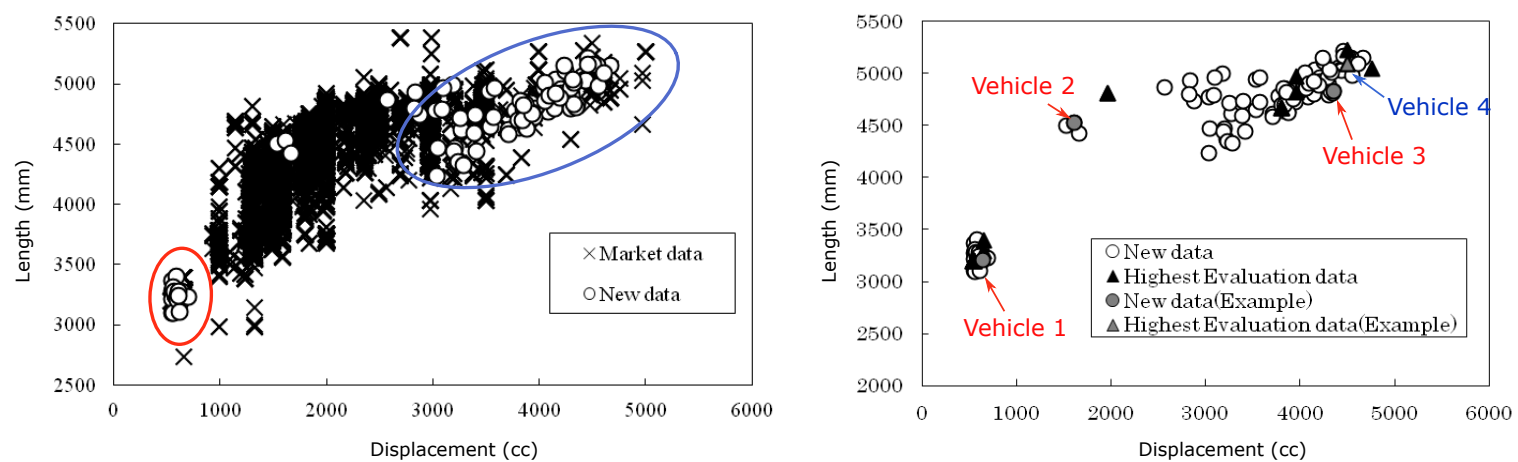

Figure 5. Relationship of displacement and length (new vehicle data and highest evaluation data).

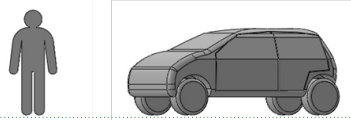

(a) Vehicle 1

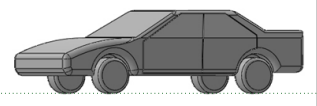

(b) Vehicle 2

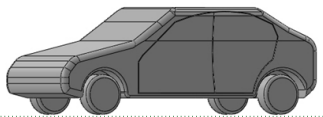

(c) Vehicle 3

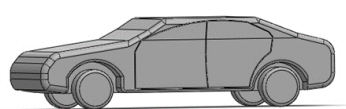

(d) Vehicle 4

Figure 6. Optimized vehicles.

Table 2. Comparison of existing vehicle and the proposed layout by our evaluation method.

\begin{tabular}{lll}
\hline Variable & Existing Vehicle & Proposed Layout \\
\hline Displacement & $4494 \mathrm{cc}$ & $4357 \mathrm{cc}$ \\
Total Length & $5090 \mathrm{~mm}$ & $4830 \mathrm{~mm}$ \\
Total Width & $1825 \mathrm{~mm}$ & $1945 \mathrm{~mm}$ \\
Total Height & $1425 \mathrm{~mm}$ & $1700 \mathrm{~mm}$ \\
\hline
\end{tabular}

\subsection{Optimization result}

In order to show the feasibility to propose new design models and the usefulness to aid in decision making, we compared the performance of our proposed method with that of the existing vehicles in the dataset as follows:

1. There are 31 vehicles with the overall DEA evaluation value of 1 in the best model of Figure 3-(c). Since DEA ratios based on Figure 3-(c) provide recommendations on the evaluation of vehicle models, we evaluated 1,200 vehicles within the upper and lower bound of vehicle variables and found 108 vehicles with DEA ratio of 1 , which is the maximum achievable ratio.

2. Figure 5 shows the relationship between Displacement and Total Length of vehicles of 108 vehicles with DEA ratio 1 (labed as New Data) and our dataset (labeled as Market Data).

By observing Figure 5, we can readily observe that our model is able to localize vehicle layouts being at the extreme frontiers of the plot (marked with ellipsoids with red and blue color in Figure 5). On the other hand, there exists few vehicles with a total Length between 3500 to $4000 \mathrm{~mm}$. This fact confirms the ability of our proposed method to localize vehicle models in the performance frontiers, which implies the usefulness to aid in the selection of vehicle layouts (consumer choice) or guiding the efforts of new vehicle development.

In order to exemplify the kind of existing vehicles and the kind of vehicles which our evaluation model is able to propose, Figure 6 shows the outline of the vehicles highlighted in Figure 5 - right column with numbers 1 to 4 , along with a male dummy model $(171 \mathrm{~cm})$. Also, Table 2 shows the comparison in variable dimensions of existing vehicles with maximum evaluation ratio. As we can observe from Figure 6 and Table 2, our proposed model eases the evaluation of the performance frontiers not only of the existing vehicle layouts, but also offer insights on the new vehicle layouts. We believe our proposed approach is useful to aid in the evaluation and proposal of product layouts. 


\section{FINAL NOTES}

In this paper, we proposed Data Envelopment Analysis with hierarchy concepts, to generate evaluation models by integrating with Interpretive Structural Modeling (ISM) and Hierarchical Clustering (HC). Here,

1. ISM constructs hierarchical digraphs (skeletons),

2. HC reduces the dimensionality of pairwise comparisons (correlations) of design variables, and suggests possible evaluation configurations, and

3. DEA computes weights suggesting optimal evaluation metrics.

Computational experiments using more than twenty thousand vehicles from 1982 to 2013 confirmed the usefulness to (1) generate the diverse vehicle evaluation metrics, and (2) to suggest optimal configurations for vehicle design layouts.

Future work aims at integrating with studies in differentiation and integration in complex mechanical products (Ishii (2017)), using nonconvex learning algorithms to sample the design search space with exploration and exploitation concepts (Parque et al. (2013); Parque and Miyashita (2015, 2017, $2018 \mathrm{a}, \mathrm{b})$ ), using succinct and canonical representation of the combinatorial search space of correlation variables to render a compact set of vehicle design variables (Parque and Miyashita (2018c,d)), and using Topology Optimization to aid towards the definition of optimal vehicle structures. Also, the feasibility of incorporating soft data as consumer satisfaction in the vehicle variables is in our agenda. We believe the introduction of hierarchy into DEA-based studies enables the construction of deeper evaluation models, which is advantageous to explore robust metrics, and thus, to tackle the challenge to evaluate complex mechanical products effectively.

\section{REFERENCES}

Barat, M., Tohidi, G. and Sanei, M. (2018). "DEA for nonhomogeneous mixed networks", Asia Pacific Management Review.

Cantner, U., Krueger, J. J. and Soellnerz, R. "Product quality, product price, and share dynamics in the German compact car market", Industrial and Corporate Change, Vol. 21, No. 5, pp. 1085-1115.

Charnes, A., Cooper, W. and Rhodes, E. (1978). "Measuring the efficiency of decision making units", European Journal of Operational Research, Vol. 2 No. 6, pp. 429-444.

Doyle, J. and Green, R. "Comparing Products using Data Envelopment Analysis", Omega, Vol. 19 No. 6, pp. $631-638$.

Fernandez-Castro, A. S. and Smith, P. C. (2002). "Lancaster's characteristics approach revisited: product selection using non-parametric methods", Managerial and Decision Economics, Vol. 23 No. 2, pp. 83-91.

Førsund, F. R. (2018). "Economic interpretations of DEA", Socio-Economic Planning Sciences, Vol. 61, pp. 9-15. Recent developments on the use of DEA in the public sector.

Gonzalez, E., Carcaba, A. and Ventura, J. "How car dealers adjust prices to reach the product efficiency frontier in the Spanish automobile market", Omega, Vol. 51, pp. 38-48.

Han, Y., Geng, Z., Gu, X. and Zhu, Q. "Energy efficiency analysis based on DEA integrated ISM: A case study for Chinese ethylene industries", Engineering Applications of Artificial Intelligence, Vol. 45, pp. 80-89.

Hsiao, S.-W., Ko, Y.-C., Lo, C.-H. and Chen, S.-H. "An ISM, DEI, and ANP based approach for product family development", Advanced Engineering Informatics, Vol. 27, No. 1, pp. 131-148.

Ishii, T., Parque, V., Miura, S., Miyashita, T. (2017). "Definition and support of differentiation and integration in mechanical structure using S-curve theory and wavelet transform", 21st International Conference on Engineering Design, Vol. 6 No. DS87-6, 2017, pp. 355-364.

Papagapiou, A., Mingers, J. and Thanassoulis, E. (1997). "Would you buy a used car with DEA?", OR Insight, Vol. 10 No. 1, Jan, pp. 13-19.

Papahristodoulou, C. "A DEA model to evaluate car efficiency", Applied Economics, Vol. 29, pp. 1493-1508.

Parque, V., Kobayashi, M., Higashi, M. "Reinforced Explorit on Optimizing Vehicle Powertrains", International Conference on Neural Information Processing, pp. 579-586.

Parque, V. and Miyashita, T. (2017). "A method to learn high-performing and novel product layouts and its application to vehicle design”, Neurocomputing, Vol. 248, pp. 41-56.

Parque, V. and Miyashita, T. (2015). "Learning the Optimal Product Design Through History", International Conference on Neural Information Processing, pp. 382-389.

Parque, V. and Miyashita, T. "On Learning Fuel Consumption Prediction in Vehicle Clusters", IEEE 42nd Annual Computer Software and Applications Conference (COMPSAC), pp. 116-121. 
Parque, V. and Miyashita, T. "On vehicle surrogate learning with genetic programming ensembles”, GECCO '18 Proceedings of the Genetic and Evolutionary Computation Conference Companion, pp. 1704-1710.

Parque, V. and Miyashita, T. "Towards the Succinct Representation of m Out of n", Internet and Distributed Computing Systems - 11th International Conference, pp. 16-26.

Parque, V. and Miyashita, T. "Unranking Combinations Using Gradient-Based Optimization”, IEEE 30th International Conference on Tools with Artificial Intelligence, pp. 579-586.

Raut, R. D., Gardas, B. B., Jha, M. K. and Priyadarshinee, P. (2017). "Examining the critical success factors of cloud computing adoption in the msmes by using ism model", The Journal of High Technology Management Research, Vol. 28 No. 2, pp. 125-141.

Seiford, L. M. and Zhu, J. (2003). "Context-dependent data envelopment analysis - measuring attractiveness and progress”, Omega, Vol. 31 No. 5, pp. 397-408.

Sueyoshi, T., Yuan, Y. and Goto, M. (2017). “A literature study for DEA applied to energy and environment”, Energy Economics, Vol. 62, pp. 104-124.

Tavana, M., Li, Z., Mobin, M., Komaki, M. and Teymourian, E. (2016). "Multi-objective control chart design optimization using NSGA-iii and MOPSO enhanced with DEA and topsis", Expert Systems with Applications, Vol. 50, pp. 17-39.

Voltes-Dorta, A., Perdiguero, J. and Jiménez, J. L. (2013). “Are car manufacturers on the way to reduce CO2 emissions?: A DEA approach', Energy Economics, Vol. 38, pp. 77-86.

Warfield, J. N. (1973a). "On arranging elements of a hierarchy in graphic form', IEEE Transactions on Systems, Man, and Cybernetics, Vol. SMC-3 No. 2, March, pp. 121-132.

Warfield, J. N. (1973b). "Binary matrices in system modeling', IEEE Transactions on Systems, Man, and Cybernetics, Vol. SMC-3 No. 5, Sept, pp. 441-449.

Warfield, J. N. (1974a). "Developing subsystem matrices in structural modeling”, IEEE Transactions on Systems, Man, and Cybernetics, Vol. SMC-4 No.1, Jan, pp. 74-80.

Warfield, J. N. (1974b). "Developing interconnection matrices in structural modeling”, IEEE Transactions on Systems, Man, and Cybernetics, Vol. SMC-4 No.1, Jan, pp. 81-87.

Warfield, J. N. (1973c). "Constructing operational value systems for proposed two-unit coalitions", IEEE Conference on Decision and Control, pp. 204-213.

Warfield, J. N. (1974). "Toward interpretation of complex structural models", IEEE Transactions on Systems, Man, and Cybernetics, Vol. SMC-4 No. 5, Sept, pp. 405-417. 